\title{
Review Article \\ Does Restenosis Still Hamper the Benefit of Carotid Artery Revascularization?
}

\author{
C. J. de Witte, G. W. van Lammeren, F. L. Moll, and G. J. de Borst \\ Department of Vascular Surgery, University Medical Center Utrecht, P.O. Box 85500, 3508 GA Utrecht, The Netherlands \\ Correspondence should be addressed to G. J. de Borst; g.j.deborst-2@umcutrecht.nl
}

Received 29 November 2012; Accepted 13 January 2013

Academic Editors: A. Csaszar and C.-C. Wu

Copyright (C) 2013 C. J. de Witte et al. This is an open access article distributed under the Creative Commons Attribution License, which permits unrestricted use, distribution, and reproduction in any medium, provided the original work is properly cited.

\begin{abstract}
Both carotid endarterectomy (CEA) and carotid artery angioplasty with stenting (CAS) may offer acceptable short-term results in symptomatic or asymptomatic patients with carotid stenosis. Independent on the type of revascularization, the long-term benefit may be limited by recurrent stenosis, especially after endovascular treatment. Pathophysiological studies suggest that atherosclerotic plaque composition is an independent predictor of restenosis. Identification of certain plaque characteristics could help risk stratify patients in order to decide on the best therapy and minimize the risk of restenosis. Although currently no gold standard exists for the approach of recurrent carotid stenosis, both redo CEA and CAS seem safe therapeutic options. Limited data are available on treatment of recurrent carotid in-stent stenosis. More data are required in order to recommend the best therapy for in-stent restenosis.
\end{abstract}

\section{Introduction}

Patients with symptomatic carotid atherosclerotic disease with a significant degree of stenosis require carotid revascularization to prevent future major ipsilateral stroke. Currently, the gold standard therapy is still carotid endarterectomy (CEA), but carotid artery angioplasty with stenting (CAS) has gained interest over the past decades due to its minimal invasive character. However, in terms of prevention of procedural related stroke, in all randomized trials comparing CEA with CAS for both symptomatic and asymptomatic patients, surgery was superior over endovascular treatment [1-3].

Independent on the type of revascularization, periprocedural complications have decreased over time resulting in a clear short-term benefit in terms of stroke prevention from uncomplicated revascularization as compared to best medical treatment. However, in the long term, the benefit of revascularization is still hampered by the occurrence of restenosis which is associated with a modestly increased risk of stroke [4]. Again, durability in terms of freedom from restenosis following revascularization is higher after surgery as compared to CAS. However, still no consensus exists on the treatment algorithm of carotid restenosis following carotid revascularization.
The present review will address the pathophysiology, risk factors, diagnosis, and management of restenosis after carotid revascularization. Different aspects of carotid surgery and stenting as primary treatment for carotid artery disease will be discussed with respect to restenosis, and the treatment of restenotic lesions will also be addressed. In addition, the role of the underlying plaque composition and the interrelation with patient characteristics and its impact on restenosis rates will be discussed.

\section{Definition}

Restenosis is generally defined as $>50 \%$ recurrent luminal narrowing as detected on Duplex Ultrasound (DUS), Computed Tomographic Angiography (CTA), Magnetic Resonance Angiography (MRA), or by conventional angiography. Restenosis $>70 \%$ generally is classified as severe restenosis.

The degree of stenosis can be assessed most accurately by conventional angiography. The European Carotid Surgery Trial (ECST) [5] and the North American Symptomatic Carotid Endarterectomy Trial (NASCET) $[6,7]$ have reported different methods to assess the degree of stenosis. A schematic overview of the differences is depicted in Figure 1. It is important to realize that a degree of stenosis of $70-99 \%$ 


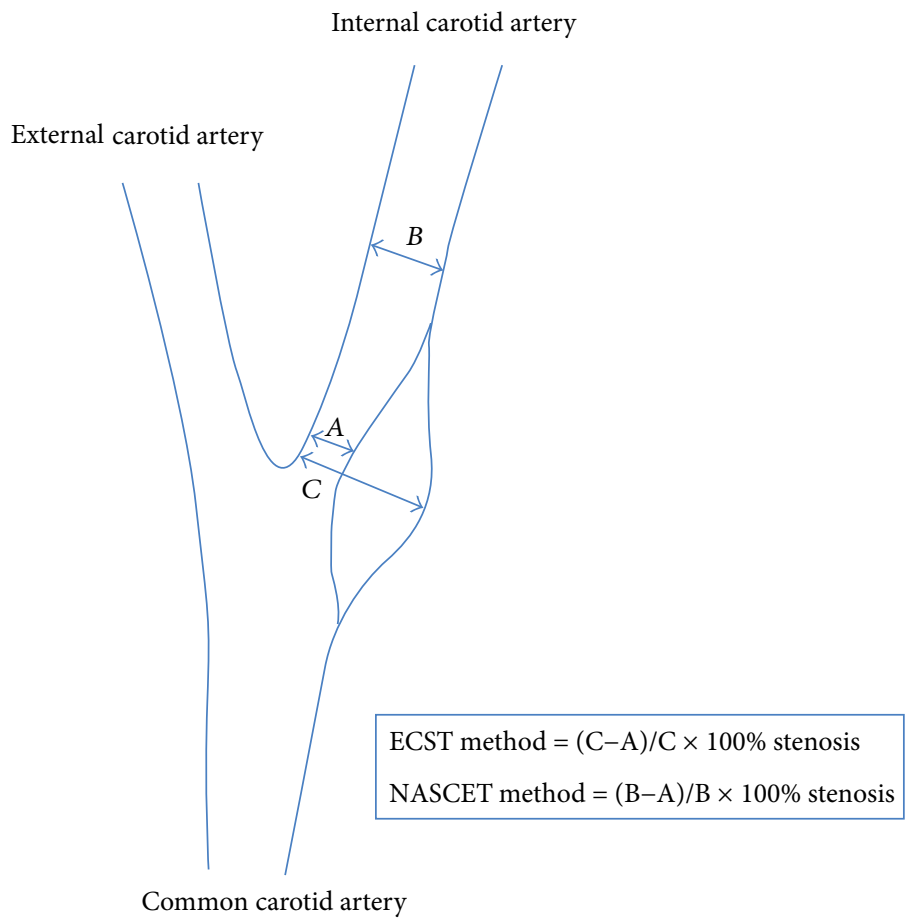

FIGURE 1: Methods to assess the degree of stenosis using conventional angiography according to the ECST method $((\mathrm{C}-\mathrm{A}) / \mathrm{C} \times 100 \%)$ and the NASCET method $((\mathrm{B}-\mathrm{A}) / \mathrm{B} \times 100 \%)$.

measured according to the NASCET criteria corresponds to a degree of stenosis of $80-99 \%$ according to the ECST criteria. A $50-70 \%$ stenosis according to the NASCET method corresponds to $70-79 \%$ stenosis according to the ECST method [8].

Despite the accuracy of angiographic measurement methods, less invasive Duplex Ultrasound scanning remains the most frequently used diagnostic modality in daily practice, especially for screening issues. DUS can be used to detect the carotid artery flow velocity to estimate the degree of stenosis, according to the modified Strandness criteria [9].

\section{Epidemiology}

Restenosis after previous CEA has been detected with increasing frequency because of the use of noninvasive testing with Duplex Ultrasound. Symptomatic recurrent stenosis has been reported to range from 0.65 to $3.6 \%$ and asymptomatic recurrent stenosis, based on noninvasive imaging studies, from 8.8 to $19 \%$ depending on the time interval defined $[4,10-$ 14]. Risk factors for restenosis are diverse and consist of smoking, elevated cholesterol, female sex, young age $(<65)$, previous ipsilateral carotid surgery, and diabetes. Multiple studies have reported about different risk factors. However, the predictive value of individual risk factors in the occurrence and degree of restenosis is limited.

Several randomised multicenter trials comparing outcome from CEA versus CAS provided information on the occurrence of restenosis after initial treatment $[15,16]$. The Carotid and Vertebral Artery Transluminal Angioplasty
Study (CAVATAS) revealed a three times higher incidence of relevant restenosis $(>70 \%)$ after endovascular treatment compared with endarterectomy [15]. However, the 50 patients in the endovascular arm who underwent additional stenting had a significant lower risk of restenosis compared to the 145 patients who received balloon angioplasty (PTA) alone. After CAVATAS primary balloon angioplasty alone for carotid intervention was virtually abandoned and generally replaced by primary stenting.

In the Stent-Protected Angioplasty versus CarotidEndarterectomy trial (SPACE), an $11 \%$ incidence of severe restenosis $(>70 \%)$ was found in the stenting group, versus a $5 \%$ incidence in the endarterectomy group, at two years after intervention as assessed with DUS [16]. On the other hand, a subanalysis in the Carotid Revascularization Endarterectomy versus Stenting Trial (CREST), conducted among 2191 symptomatic patients, showed no differences in restenosis rate $(>70 \%)$ at two years between carotid endarterectomy and stenting (5,8\%), as detected by DUS. In the Endarterectomy Versus Angioplasty in patients with Severe Symptomatic Carotid Stenosis (EVA3-S) trial, moderate restenosis (cumulative three-year rate $12.5 \%$ versus $5.0 \%$ ) occurred significantly more often among patients treated by CAS than by CEA, but no significant difference was observed for severe restenosis [17].

Median follow-up duration ranged from two to four years after revascularization in EVA3-S, SPACE, and CREST, so firm conclusions concerning long-term patency cannot be drawn from these trials. ICSS completed the 10-year followup at the end of 2011, so their results on carotid patency after CEA and CAS will have to be awaited. 


\section{Pathophysiology}

Studies on atherosclerotic plaque composition have provided insight into pathological processes involved in primary causes of cerebrovascular symptoms, but also in local outcome and restenosis after CEA. The Athero-Express combines carotid plaque characteristics with development of restenosis by prospectively monitoring patients [18].

During plaque removal from the carotid artery, surgical trauma is inflicted on the vessel wall, resulting in local inflammation and vessel repair directly after surgery. Intimal hyperplasia and smooth muscle cell growth, or thrombus formation can result in early recurrent stenosis at the site of surgery. Late restenosis developing over two years after CEA is ascribed to renewed atherosclerotic disease. However the two processes are still not fully understood and the difference between them might be of less significance than previously believed [19].

In an Athero-Express based study, $17 \%$ of the 500 patients who underwent CEA developed restenosis $\geq 50 \%$ after one year [20]. After histological assessment of the plaque it was concluded that patients having an unstable plaque with a large lipid core and macrophage infiltration had a lower risk of developing moderate and severe restenosis. Patients with fibrous and noninflammatory plaques, on the other hand, had a higher prevalence of restenosis after CEA. The results of this study suggest that plaque composition is an independent predictor of restenosis after carotid endarterectomy.

These findings have been extrapolated to clinical presentation and surgical timing by Van Lammeren et al.

It has been established that asymptomatic patients have relatively stable plaque characteristics [21, 22]. After one year, restenosis rates were compared between asymptomatic and symptomatic patient groups. Asymptomatic patients had a higher risk of restenosis compared to patients who experienced stroke or transient ischemic attack [23].

The restenosis rates were also compared between patients who underwent CEA shortly after their last event, and patients who were operated on longer after their event. Since a carotid plaque stabilizes over time after an event and patients with a stable plaque have a higher risk of restenosis, it was hypothesized that the interval between the event and intervention influences the rate of restenosis. Biobank research confirmed that the plaques of patients treated with CEA relatively long after a cerebrovascular event presented more stable plaque characteristics, including more smooth muscle cells and less macrophages and local inflammation. A significantly decreased rate of restenosis was observed in stroke patients who were operated on within 30 days. This decrease was ascribed to plaque stabilization over time. These observations stress the importance of early revascularization after stroke to prevent restenosis in addition to the prevention of ipsilateral stroke [23]. This association was not observed for patients who suffered from a Transient Ischemic Attack (TIA), probably because the process of plaque repair and stabilization is of less significance in TIA patients, as compared to stroke patients.

If restenosis occurs, secondary interventions and redo CEA might be indicated. Longitudinal biobank research allowed histological analysis of restenotic plaques that were harvested during redo CEA. Restenotic plaques were investigated in relation to recurrence interval, and whether patient suffered from renewed cerebrovascular symptoms [24]. Results showed that early restenotic plaques contained an accumulation of smooth muscle cells and fibrous tissue, whereas late restenotic plaques demonstrated increased macrophage infiltration, calcification and a lipid core, resembling primary plaques. Patients with symptomatic restenosis had plaques with higher macrophage infiltration and a larger lipid core than asymptomatic patients, independent of recurrence interval.

In patients undergoing CAS there is still little known about associations between underlying carotid plaque characteristics and restenosis. However, research in the field of cardiology and coronary artery stenting has shown that stent thrombosis and restenosis are associated with underlying coronary plaque composition. Intracoronary stenting of vulnerable, lipid-rich lesions was associated with inadequate stent apposition and intracoronary thrombus formation during followup [25]. Lipid-rich plaques are considered to be relatively avascular and contain less cells, which is detrimental for cell proliferation and proper strut endothelialization after stent placement [26]. As for carotid stents, Ball et al. reported a case with embolic stroke three years after carotid artery stenting and showed that plaque ulceration at the location of uncovered stent struts was colocalized with thrombus formation [27]. This confirms that also after a long term, carotid stents can give rise to renewed thrombosis and cerebrovascular symptoms due to suboptimal strut endothelialization and renewed thrombus formation.

The impact of plaque composition on the occurrence of restenosis after carotid revascularization might have future implications due to developments in carotid plaque imaging. Identification of high risk individuals and high risk plaques might help to reduce restenosis rates after surgery and stenting.

\section{Diagnostics of Restenosis}

Application of standardised Duplex Ultrasound (DUS) velocity criteria for detection of stenosis severity is a controversial topic [28]. Stent placement causes anatomical and haemodynamic alterations. It is commonly accepted that velocity rates change after stent placement and new guidelines need to be developed for duplex scanning in stented patients. The stent itself may cause an increase in duplexacquired velocity measurements in the absence of technical error, residual stenotic disease, or myointimal thickening in the stent. The potential risk of using generally accepted DUS criteria for followup after CEA is an overestimation of the degree of restenosis after CAS. To complicate the interpretation of DUS after CAS even more, stent-related alterations in duplex measured velocities have been shown to be stent type dependent, and do not justify a general approach to new velocity criteria indiscriminately applied to all stents. The mechanism, magnitude, and significance of these alterations are ill-defined and emphasize the need for 
every vascular laboratory to develop specific velocity criteria for the evaluation and followup of patients after carotid stenting.

\section{Treatment}

There is currently no gold standard for the approach of carotid restenosis. The optimal therapeutic option still has to be defined and individualized. The treatment of choice therefore depends on primary treatment, as well as on patient characteristics.

It is generally accepted that patients with symptomatic restenosis require short-term revascularization. The optimal timing of intervention in asymptomatic patients with restenosis is more complex. It is difficult to predict if and when carotid stenosis will become symptomatic. Prevention of future cerebrovascular events has to be balanced with the risks of procedural complications in redo surgery or endovascular treatment.

In primary treatment of carotid stenosis by CEA, the atheromatous intimal layer of the target vessel is excised. When restenosis occurs, the treatment of choice depends on the local effects of the primary surgery and the physical condition of the patient. Redo CEA is considered a safe and reliable procedure when performed in experienced hands [4]. CAS can provide a noninvasive alternative thereby decreasing the risk of local surgical complications such as cranial nerve injury, particularly in patients with deemed increased surgical risk. The real debate on the definition which patient qualifies for high surgical risk, however, is still ongoing [29].

Currently there is no consensus on the treatment of instent restenosis. Therapeutic options vary from repeat balloon angioplasty and repeat CAS placement to CEA with stent removal, carotid artery bypass, and brachytherapy [30, 31]. CEA after CAS, although feared by some authors, has been performed with outcome comparable to primary CEA and seems durable in terms of stroke prevention and recurrent restenosis. The safety and success of available endovascular treatments for in-stent restenosis were compared with surgery in a review by Van Haaften et al. [31]. Overall it seemed that secondary CEA as well as repeat balloon angioplasty or repeat CAS placement are all safe therapeutic options. However, despite the fact that various treatments of in-stent restenosis have proven to be safe in the short term, it is important to realize that restenosis appears to be an ongoing process. Especially the patency of endovascular procedures might be hampered by this process. Due to the small study populations and the limited follow-up time, more research is required in order to recommend the best therapy for in-stent restenosis.

\section{Conclusion}

The long-term durability of carotid revascularization is hampered by restenosis. The incidence of symptomatic restenosis is low. However, timing secondary interventions to prevent future cerebrovascular events caused by restenosis remains challenging. Both surgical and endovascular interventions offer safe short-term results but it seems that secondary carotid artery stenting is associated with a higher risk of restenosis. Since no general approach has been validated, the management of recurrent stenosis should be based on a personalized approach and be discussed in a multidisciplinary forum.

Underlying carotid plaque characteristics are associated with the occurrence of restenosis after treatment and therefore contain useful prognostic information for future local patency. Current developments in carotid plaque imaging might help to risk stratify individuals at risk for restenosis in the future.

\section{References}

[1] T. G. Brott, R. W. Hobson 2nd, G. Howard et al., "Stenting versus endarterectomy for treatment of carotid-artery stenosis," The New England Journal of Medicine, vol. 363, no. 1, pp. 11-23, 2010.

[2] J. L. Mas, L. Trinquart, D. Leys et al., "Endarterectomy Versus Angioplasty in patients with Symptomatic Severe Carotid Stenosis (EVA-3S) trial: results up to 4 years from a randomised, multicentre trial," The Lancet Neurology, vol. 7, no. 10, pp. 885892, 2008.

[3] J. Ederle, J. Dobson, R. L. Featherstone, L. H. Bonati, H. B. van der Worp, and G. J. de Borst, "Carotid artery stenting compared with endarterectomy in patients with symptomatic carotid stenosis (International Carotid Stenting Study): an interim analysis of a randomised controlled trial," The Lancet, vol. 375, no. 9719, pp. 985-997, 2010.

[4] G. J. de Borst, P. Zanen, J. P. de Vries, E. D. van de Pavoordt, R. G. Ackerstaff, and F. L. Moll, "Durability of surgery for restenosis after carotid endarterectomy," Journal of Vascular Surgery, vol. 47, no. 2, pp. 363-371, 2008.

[5] C. Warlow, B. Farrell, A. Fraser, P. Sandercock, and J. Slattery, "Randomised trial of endarterectomy for recently symptomatic carotid stenosis: final results of the MRC European Carotid Surgery Trial (ECST)," The Lancet, vol. 351, no. 9113, pp. 13791387, 1998.

[6] H. J. M. Barnett, "North American symptomatic carotid endarterectomy trial: methods, patient characteristics, and progress," Stroke, vol. 22, no. 6, pp. 711-720, 1991.

[7] D. W. Taylor and H. J. M. Barnett, "Beneficial effect of carotid endarterectomy in symptomatic patients with highgrade carotid stenosis," The New England Journal of Medicine, vol. 325, no. 7, pp. 445-453, 1991.

[8] P. M. Rothwell, R. J. Gibson, J. Slattery, R. J. Sellar, and C. P. Warlow, "Equivalence of measurements of carotid stenosis: a comparison of three methods on 1001 angiograms," Stroke, vol. 25, no. 12, pp. 2435-2439, 1994.

[9] E. G. Grant, C. B. Benson, G. L. Moneta et al., "Carotid artery stenosis: gay-scale and doppler US diagnosis - society of radiologists in ultrasound consensus conference," Radiology, vol. 229, no. 2, pp. 340-346, 2003.

[10] G. M. LaMuraglia, M. C. Stoner, D. C. Brewster et al., "Determinants of carotid endarterectomy anatomic durability: effects of serum lipids and lipid-lowering drugs," Journal of Vascular Surgery, vol. 41, no. 5, pp. 762-768, 2005.

[11] J. S. Ladowski, L. M. Shinabery, D. Peterson, A. C. Peterson, and W. P. Deschner, "Factors contributing to recurrent carotid disease following carotid endarterectomy," American Journal of Surgery, vol. 174, no. 2, pp. 118-120, 1997. 
[12] D. A. Healy, R. E. Zierler, S. C. Nicholls et al., "Long-term follow-up and clinical outcome of carotid restenosis," Journal of Vascular Surgery, vol. 10, no. 6, pp. 662-669, 1989.

[13] T. Reina-Gutiérrez, F. J. Serrano-Hernando, L. Sánchez-Hervás, A. Ponce, M. V. de Ceniga, and A. Martín, "Recurrent carotid artery stenosis following endarterectomy: natural history and risk factors," European Journal of Vascular and Endovascular Surgery, vol. 29, no. 4, pp. 334-341, 2005.

[14] R. J. Valentine, S. I. Myers, R. T. Hagino, and G. P. Clagett, "Late outcome of patients with premature carotid atherosclerosis after carotid endarterectomy," Stroke, vol. 27, no. 9, pp. 1502-1506, 1996.

[15] L. H. Bonati, J. Ederle, D. J. McCabe et al., "Long-term risk of carotid restenosis in patients randomly assigned to endovascular treatment or endarterectomy in the Carotid and Vertebral Artery Transluminal Angioplasty Study (CAVATAS): long-term follow-up of a randomised trial," The Lancet Neurology, vol. 8, no. 10, pp. 908-917, 2009.

[16] H. H. Eckstein, P. Ringleb, J. R. Allenberg et al., "Results of the Stent-Protected Angioplasty versus Carotid Endarterectomy (SPACE) study to treat symptomatic stenoses at 2 years: a multinational, prospective, randomised trial," The Lancet Neurology, vol. 7, no. 10, pp. 893-902, 2008.

[17] C. Arquizan, L. Trinquart, P. J. Touboul et al., "Restenosis is more frequent after carotid stenting than after endarterectomy: the EVA-3S study," Stroke, vol. 42, no. 4, pp. 1015-1020, 2011.

[18] B. A. N. Verhoeven, E. Velema, A. H. Schoneveld et al., "Athero-express: differential atherosclerotic plaque expression of mRNA and protein in relation to cardiovascular events and patient characteristics. Rationale and design," European Journal of Epidemiology, vol. 19, no. 12, pp. 1127-1133, 2004.

[19] G. J. De Borst and F. Moll, "Biology and treatment of recurrent carotid stenosis," The Journal of Cardiovascular Surgery, vol. 53, no. 1, Supplement 1, pp. 27-34, 2012.

[20] W. E. Hellings, F. L. Moll, J. P. P. M. De Vries et al., "Atherosclerotic plaque composition and occurrence of restenosis after carotid endarterectomy," Journal of the American Medical Association, vol. 299, no. 5, pp. 547-554, 2008.

[21] W. Peeters, W. E. Hellings, D. P. V. De Kleijn et al., "Carotid atherosclerotic plaques stabilize after stroke insights into the natural process of atherosclerotic plaque stabilization," Arteriosclerosis, Thrombosis, and Vascular Biology, vol. 29, no. 1, pp. 128-133, 2009.

[22] B. Verhoeven, W. E. Hellings, F. L. Moll et al., "Carotid atherosclerotic plaques in patients with transient ischemic attacks and stroke have unstable characteristics compared with plaques in asymptomatic and amaurosis fugax patients," Journal of Vascular Surgery, vol. 42, no. 6, pp. 1075-1081, 2005.

[23] G. W. Van Lammeren, W. Peeters, J. P. P. M. De Vries et al., "Restenosis after carotid surgery: the importance of clinical presentation and preoperative timing," Stroke, vol. 42, no. 4, pp. 965-971, 2011.

[24] W. E. Hellings, F. L. Moll, J. P. P. M. De Vries, P. De Bruin, D. P. V. De Kleijn, and G. Pasterkamp, "Histological characterization of restenotic carotid plaques in relation to recurrence interval and clinical presentation: a cohort study," Stroke, vol. 39, no. 3, pp. 1029-1032, 2008.

[25] T. Kubo, T. Imanishi, H. Kitabata et al., "Comparison of vascular response after sirolimus-eluting stent implantation between patients with unstable and stable angina pectoris. A serial optical coherence tomography study," Cardiovascular Imaging, vol. 1, no. 4, pp. 475-484, 2008.
[26] A. V. Finn, G. Nakazawa, E. Ladich, F. D. Kolodgie, and R. Virmani, "Does underlying plaque morphology play a role in vessel healing after drug-eluting stent implantation?" Cardiovascular Imaging, vol. 1, no. 4, pp. 485-488, 2008.

[27] T. C. Ball, J. R. Foerst, M. Vorpahl, R. J. Powell, R. Virmani, and A. V. Kaplan, "Embolic stroke after carotid stenting: microscopic computed tomography analysis of en bloc surgical specimen demonstrating ulceration," Circulation, vol. 121, no. 14, pp. 1661-1663, 2010.

[28] G. J. de Borst, R. Meijer, R. H. Lo, H. W. G. Vosmeer, R. G. A. Ackerstaff, and F. L. Moll, "Effect of carotid angioplasty and stenting on duplex velocity measurements in a porcine model," Journal of Endovascular Therapy, vol. 15, no. 6, pp. 672-679, 2008.

[29] M. Fokkema, A. G. den Hartog, M. L. Bots, I. van der Tweel, F. L. Moll, and G. J. de Borst, "Stenting versus surgery in patients with carotid stenosis after previous cervical radiation therapy: systematic review and meta-analysis," Stroke, vol. 43, no. 3, pp. 793-801, 2012.

[30] A. Gonzalez, M. Drummond, S. McCord, and H. E. Garrett Jr., "Carotid endarterectomy for treatment of in-stent restenosis," Journal of Vascular Surgery, vol. 54, no. 4, pp. 1167-1169, 2011.

[31] A. C. Van Haaften, M. L. Bots, F. L. Moll, and G. J. De Borst, "Therapeutic options for carotid in-stent restenosis: review of the literature," Journal of Vascular and Interventional Radiology, vol. 21, no. 10, pp. 1471-1477, 2010. 


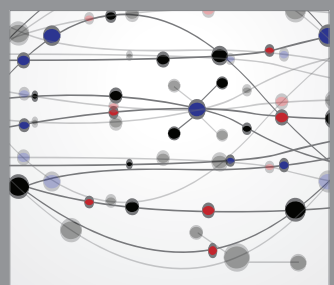

The Scientific World Journal
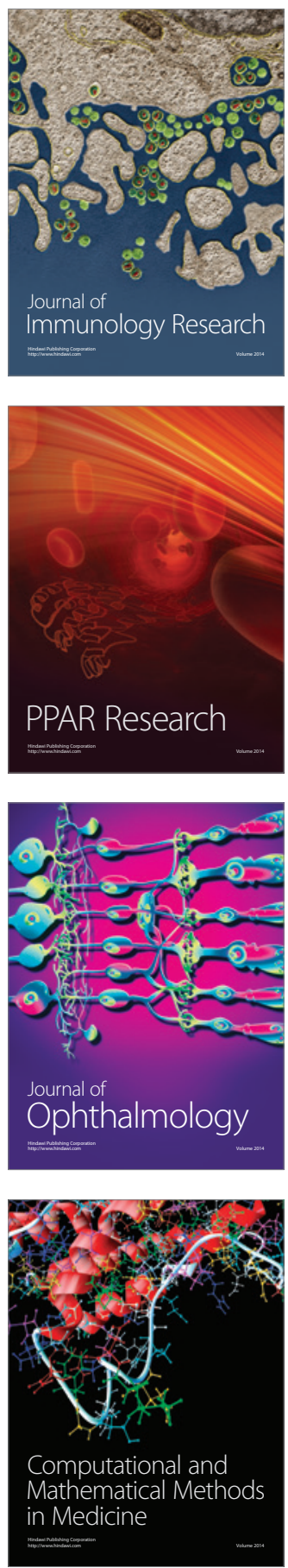

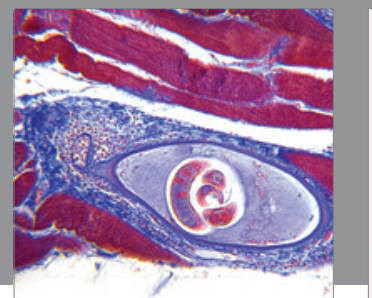

Gastroenterology

Research and Practice
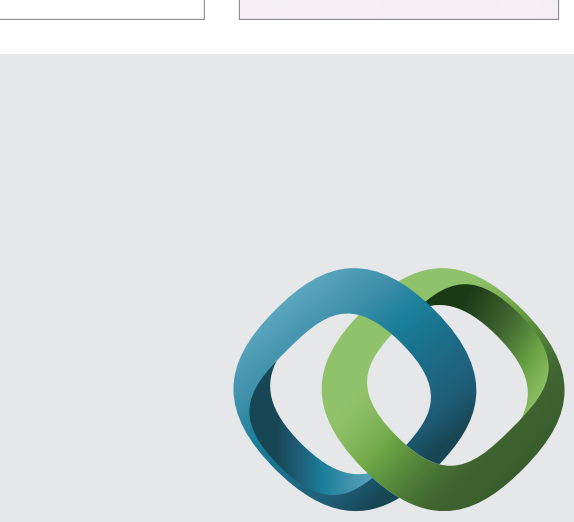

\section{Hindawi}

Submit your manuscripts at

http://www.hindawi.com
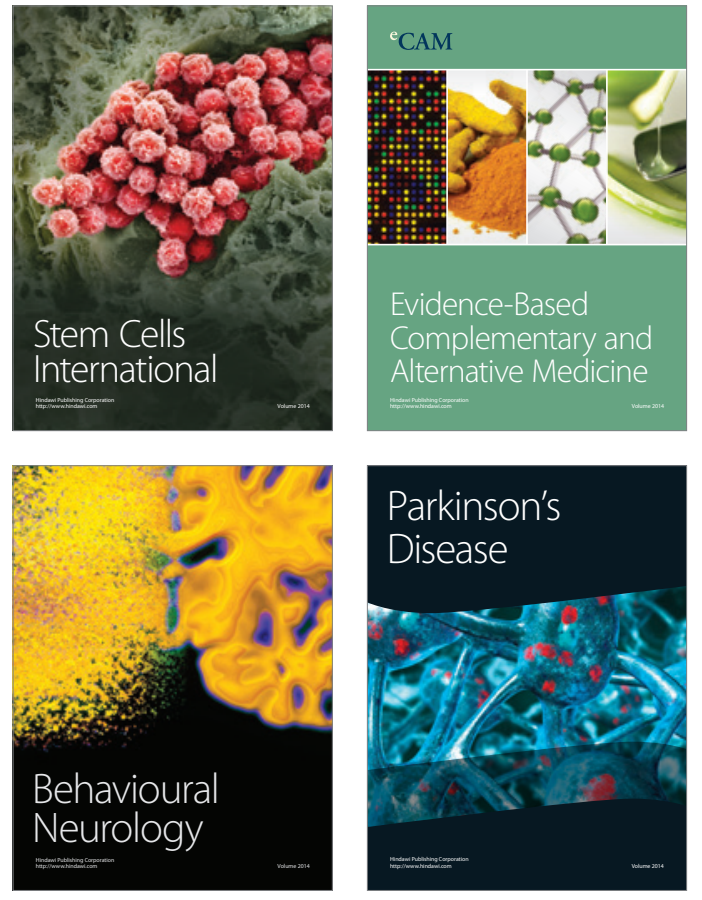
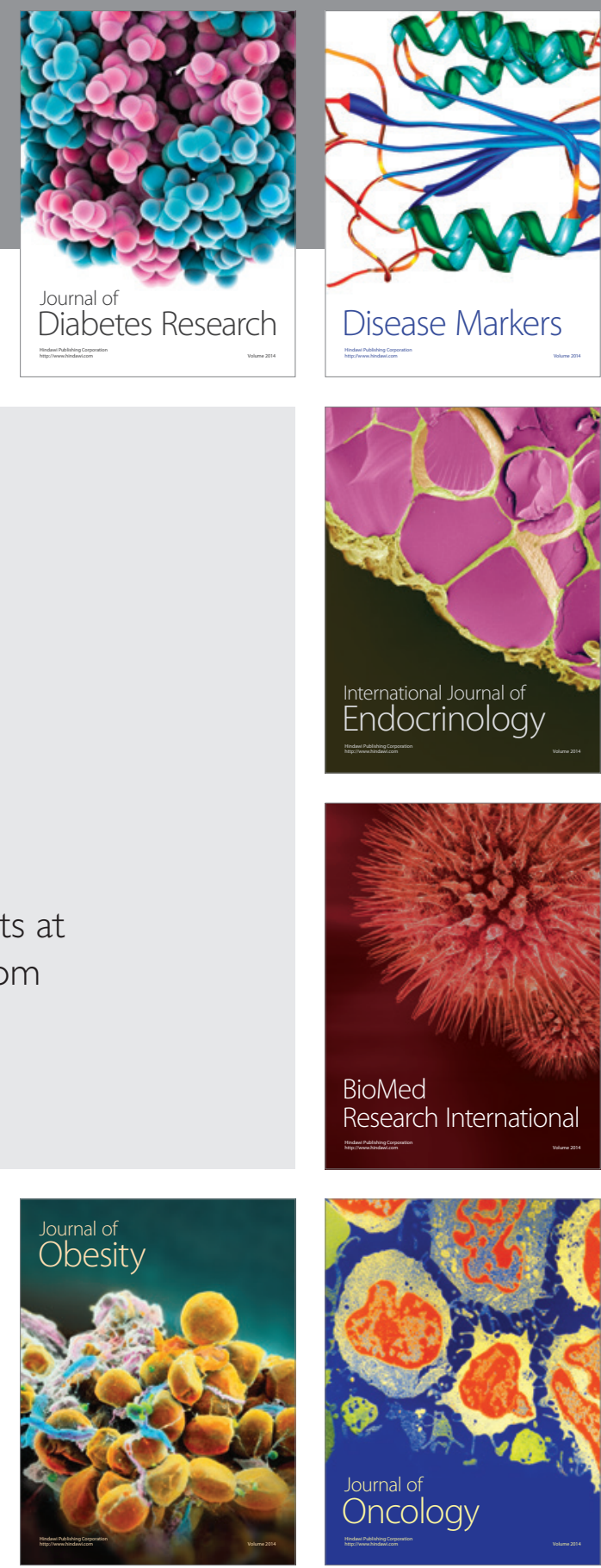

Disease Markers
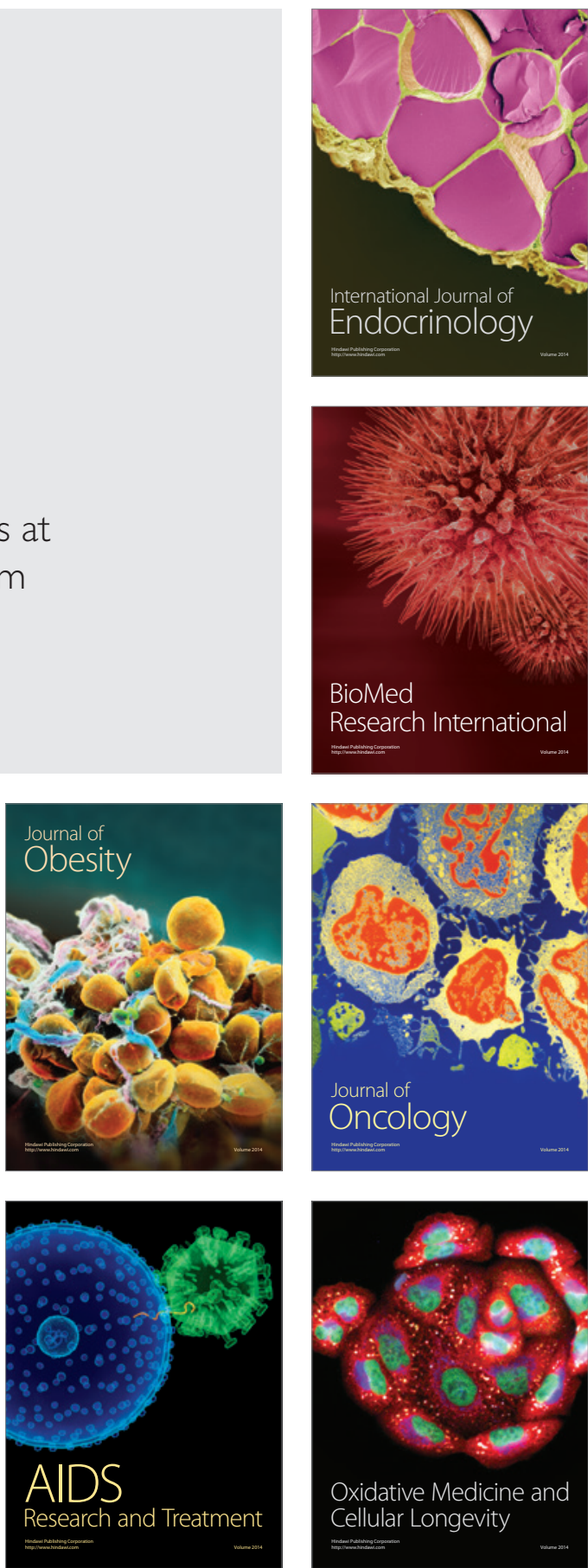